\title{
Design of Miniaturized and Polarization-Insensitive Double-Layer Frequency Selective Surface Based on Meander Lines
}

\author{
Feifei Huo ${ }^{D},{ }^{1}$ Fei Liu, ${ }^{1}$ Min Zhu, ${ }^{1}$ and Jianhui Bao ${ }^{2}$ \\ ${ }^{1}$ School of Electronic and Information Engineering, Jinling Institute of Technology, Nanjing 211169, China \\ ${ }^{2}$ School of Electronics and Information Engineering, Hebei University of Technology, Tianjin 300401, China \\ Correspondence should be addressed to Feifei Huo; huofeifei4750@jit.edu.cn
}

Received 31 August 2021; Revised 24 October 2021; Accepted 8 November 2021; Published 23 November 2021

Academic Editor: Chien-Jen Wang

Copyright (c) 2021 Feifei Huo et al. This is an open access article distributed under the Creative Commons Attribution License, which permits unrestricted use, distribution, and reproduction in any medium, provided the original work is properly cited.

The work presented in this paper concerns a method for the miniaturized frequency selective surface (FSS) based on the meander lines. A miniaturized dual-bandstop FSS structure based on meander lines with spiral-shape is proposed and simulated. The equivalent circuit and current distributions are introduced to explain the FSS performance. The size of the unit cell is $10 \mathrm{~mm}$, which is about 0.037 wavelength at the first resonant frequency. Simulation results indicate that the proposed FSS has a frequency shift smaller than $1 \%$ for different polarizations with an oblique incident angle of $60^{\circ}$. A prototype of the FSS is fabricated and measured. The measurement results show that the FSS is polarization-insensitive and angle-insensitive.

\section{Introduction}

In the past few years, frequency selective surfaces (FSSs) have been used for a variety of applications, including absorbers $[1,2]$, radar stealth [3-5], communications [6-8], and other aspect [9-11] in the microwave, millimeter wave, and infrared wave. FSS is a two-dimensional periodic structure which consists of metallic patches or apertures etched on a dielectric substrate [12]. With the increasing requirement of FSS technology, the traditional FSS cannot satisfy the practical application because the large unit size will lead to the premature production of the grating lobe when the elements resonant. Another reason is that traditional FSS is difficult to be applied in a limited space area or on irregular surfaces.

Miniaturized FSS can be used to overcome the above difficulties. Many methods are employed to achieve the purpose of unit size reduction, including the coupling technology based on the capacitive surface and inductive surface [13-15], loading with passive lumped elements (capacitors and inductors) $[16,17]$, and the unit curling and interdigitating design [18-23]. In [18], a convoluted structure is used for miniaturized FSS, and the element size is about 0.08 wavelength of the free-space. In [19], a miniaturized FSS is single layer and composed of the Archimedean spiral type with the interdigital capacitance between the corners of metallic patches, and the element size is about 0.066 wavelength at the resonant frequency. In [20], a miniaturized dual-band FSS with closely spaced resonance is proposed. The proposed FSS comprises meander lines printed on a single-layer dielectric substrate, and the element size is about 0.065 wavelength at the resonant frequency. In [21], a miniaturized FSS is proposed that could fulfill thin substrate design for curved application and provide stable resonant frequency. The compact unit size can achieve 0.052 wavelength at the lower resonant frequency. In [22], a miniaturized dual-band FSS is designed by using branched tortuous pattern of cross-dipole element backed by a wire grid of the same periodicity. The unit size of the proposed FSS is about 0.075 wavelength at the first resonant frequency.

In this paper, a miniaturized dual-bandstop FSS with meander lines is proposed. The structure and the equivalent circuit model (ECM) of the unit cell are introduced and analyzed. The unit cell size can achieve as small as 0.037 wavelength at the first resonant frequency. The frequency response under different polarizations and angles of the incidence plane wave of the proposed FSS is simulated. A prototype of the FSS is fabricated and measured, and the measurement results indicate that the proposed FSS is polarization-insensitive and angle-insensitive. 


\section{Unit Cell Design and Simulation Results}

2.1. Unit Cell Design. The evolution process of the miniaturized FSS unit cell is shown in Figure 1. A meander-line FSS is a planar-periodic structure, and the parameters of a unit cell are detailed in Figure 1(a). The meander-line FSS is imprinted on a standard $1 \mathrm{~mm}$ thick FR-4 substrate $\left(\varepsilon_{r}=4.4 \tan \delta=0.02\right)$. According to the equivalent circuit method of the FSS, the meander-line FSS can be equivalent to a series circuit with inductance when a plane wave propagates along the $z$-direction with the electric-field component polarized along the $y$-axis (TE polarization) and a parallel resonant circuit when the incident wave propagates along the $z$-direction with the electric-field component polarized along the $x$-axis (TM polarization). The series circuit means that the meander-line is a low-pass filter for TE mode, and the parallel circuit means that the meander-line FSS is a bandstop filter for TM mode.

A single-layer FSS can be obtained based on the meander-line FSS. The configuration of the single-layer FSS unit cell is shown in Figure 1(b), it can be observed that the unit cell is spiral-shaped, and every unit cell contains four meander-line structures. The parameters of the FSS are $l=4.6 \mathrm{~mm}, w=0.3 \mathrm{~mm}, w s=0.7 \mathrm{~mm}, p=10 \mathrm{~mm}$, and $t=1 \mathrm{~mm}$, respectively. Figure 2 (a) shows the topology of the ECM of the single-layer FSS unit cell. The ECM is a serial $L C$ resonator, where $Z_{0}=377 \Omega$ is the wave impedance of the free-space. The inductance $L$ is produced by the metallic strips, and the capacitance $C$ is produced by the coupling between the metallic strips. The approximate relationship between the values of the circuit parameters and the proposed FSS parameters is provided in [24] as follows:

$$
\left\{\begin{array}{l}
C \propto \varepsilon_{0}\left(1+\varepsilon_{r}\right) \frac{L_{\text {slot }}}{\pi} \ln \left(\frac{1}{\sin \left(\pi g / 2 L_{\text {slot }}\right)}\right), \\
L \propto \mu_{0} \frac{L_{\text {line }}}{2 \pi} \ln \left(\frac{1}{\sin \left(\pi w / 2 L_{\text {line }}\right)}\right),
\end{array}\right.
$$

where $L_{\text {line }}$ is the length of the meander lines and $L_{\text {slot }}$ is the length of the meander slots.

In order to further miniaturize the unit cell, double-layer FSS structure is utilized. The structure on the upper and bottom surfaces of the substrate is spiral-shaped and connected through the vias, as shown in Figure 1(c). The ECM of the double-layer FSS is shown in Figure 2(b), which is composed of two shunt serial $L C$ resonators $\left(L_{1}-C_{1}\right.$ and $L_{2}$ $C_{2}$ ) separated by a short transmission line $Z_{\mathrm{h}}$ with a serial inductor $L_{3} . Z_{\mathrm{h}}$ can be ignored in this design when the transmission line is very short. The equivalent impedance $Z_{\mathrm{FSS}}$ of the ECM can be found as follows:

$$
Z_{\mathrm{FSS}}=\frac{\left(j \omega L_{1}+\left(1 / j \omega C_{1}\right)\right)\left(j \omega L_{2}+j \omega L_{3}+\left(1 / j \omega C_{2}\right)\right)}{j \omega\left(L_{1}+L_{2}+L_{3}\right)+\left(1 / j \omega C_{1}\right)+\left(1 / j \omega C_{2}\right)},
$$

where $Z_{\mathrm{FSS}}$ is the equivalent impedance of the ECM. According to the theory of transmission lines, the transmission coefficient of the proposed ECM can be calculated by

$$
T(\omega)=\frac{2 Z_{\mathrm{FSS}}}{2 Z_{\mathrm{FSS}}+Z_{0}} .
$$

It is easy to find that two transmission zeroes occur while the impedance of the equivalent circuit $Z_{\mathrm{FSS}}=0 \Omega$ and one transmission pole occurs while the impedance of the equivalent circuit $Z_{\mathrm{FSS}}=\infty$. From equation (2), the frequencies of the zeroes and pole can be determined as follows:

$$
\left\{\begin{array}{l}
\omega_{\text {zero }}^{1}=\frac{1}{\sqrt{L_{1} C_{1}}}, \\
\omega_{\text {zero }}^{2}=\frac{1}{\sqrt{\left(L_{2}+L_{3}\right) C_{2}}}, \\
\omega_{\text {pole }}=\sqrt{\left(C_{1}+C_{2}\right) /\left(L_{1}+L_{2}+L_{3}\right) C_{1} C_{2}} .
\end{array}\right.
$$

As a result, the lumped parameters of the equivalent circuit proposed in Figure 2(b) are calculated as $L_{1}=3.6 \mathrm{nH}$, $C_{1}=1.1 \mathrm{pF}, \quad L_{2}=10 \mathrm{nH}, \quad C_{2}=1.3 \mathrm{pF}, \quad$ and $\quad L_{3}=5.5 \mathrm{nH}$, respectively.

The double-layer FSS unit cell is displayed in Figure 3. It can be seen that the upper layer of the unit cell consists of two spirally arranged structures as shown in Figure 3(a), and the bottom layer which has the same structure is orthogonal to the upper layer as shown in Figure 3(b). There are 4 vias shown in Figure 3(c) used to connect the upper and bottom layers.

The inductances $\mathrm{L}_{1}$ and $L_{2}$ are produced by the metallic strips of the upper layer and bottom layers, respectively. The values of $L_{1}$ and $L_{2}$ are proportional to the length of the metallic strips and inversely proportional to the width of the strips. The capacitances $C_{1}$ and $C_{2}$ are produced by the coupling between the metallic strips of the upper and the bottom layers, respectively. The values of $C_{1}$ and $C_{2}$ are inversely proportional to the distance between metallic strips. $L_{3}$ represents the inductance of the copper vias, and the value of $L_{3}$ is proportional to the length of the via and inversely proportional to the diameter of the via. From equation (1), the parameters of the double-layer FSS unit cell are set as follows: $l_{3}=9.6 \mathrm{~mm}, l_{4}=4.65 \mathrm{~mm}$, $l_{5}=3.85 \mathrm{~mm}, w=0.3 \mathrm{~mm}, g=0.6 \mathrm{~mm}, p=10 \mathrm{~mm}$, and $t=1 \mathrm{~mm}$.

2.2. Simulation Results. The transmission coefficient of the single-layer FSS is simulated by the full-wave field solver HFSS and compared with the calculated one obtained by the ECM; the results are displayed in Figure 4(a). There is a resonant frequency at $2.6 \mathrm{GHz}$ both for ECM and simulation results as shown in Figure 4(a). The length of the unit cell is about 0.087 wavelength at $2.6 \mathrm{GHz}$.

The transmission coefficient of the double-layer FSS is also simulated and compared with the one obtained by the 


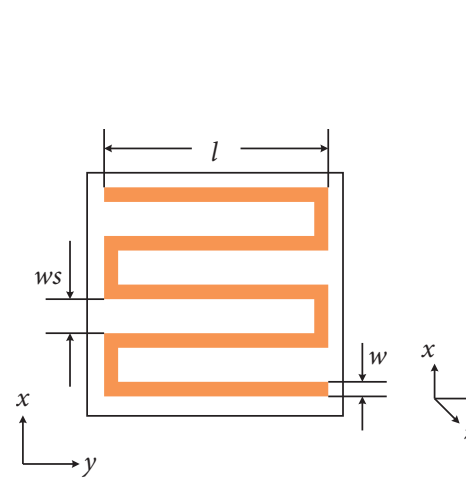

(a)

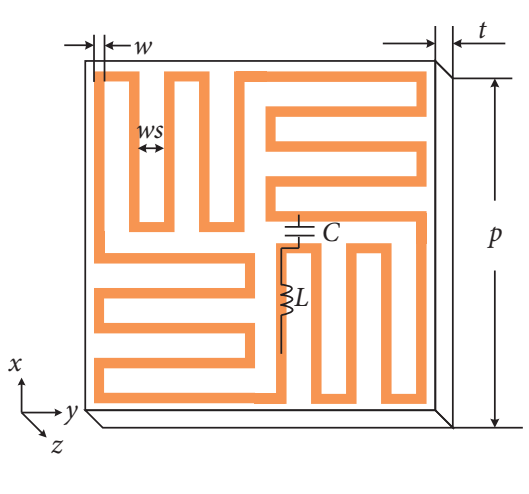

(b)

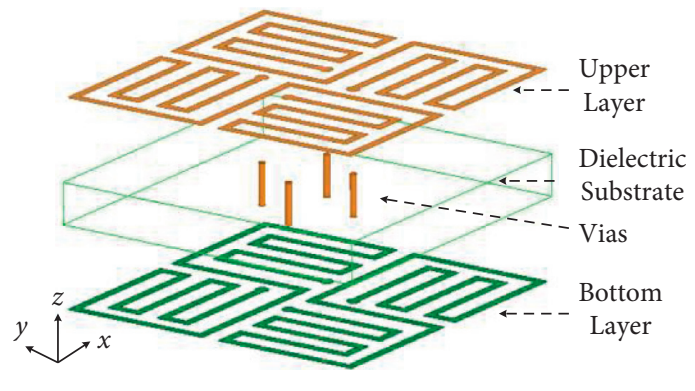

(c)

FIGURE 1: Evolution process of the polarization-insensitive and miniaturized FSS unit cell: (a) the meander-line FSS; (b) the single-layer FSS; (c) the double-layer FSS.

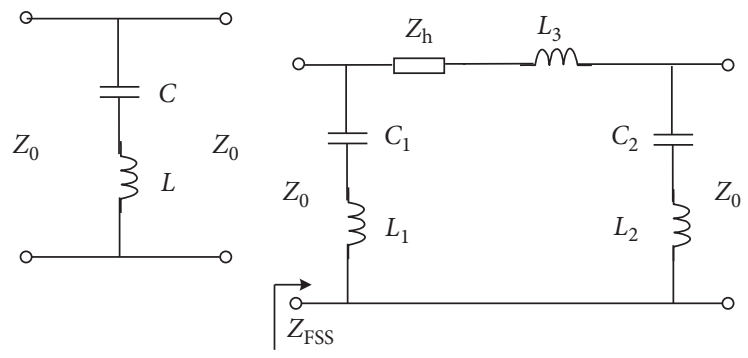

(a)

(b)

FIGURE 2: Equivalent circuit models of the FSS unit cells: (a) the single-layer FSS; (b) the double-layer FSS.

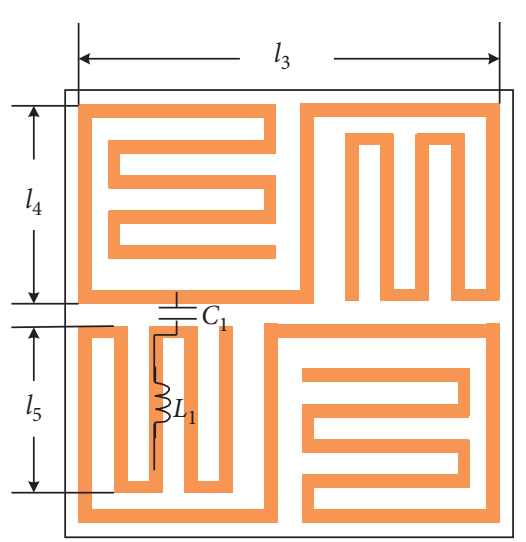

(a)

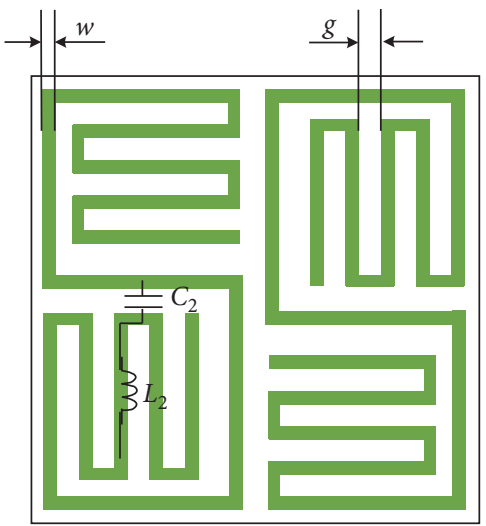

(b)

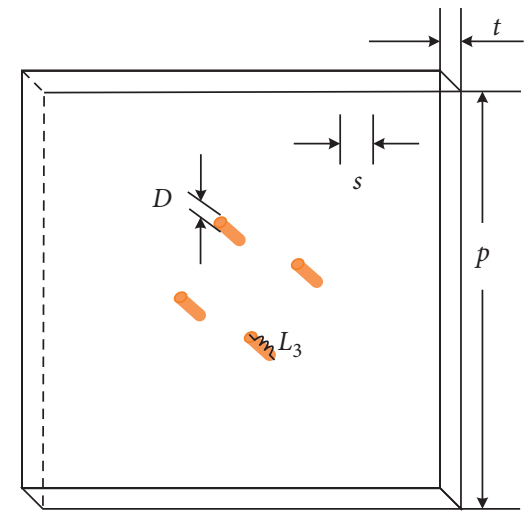

(c)

FIgURE 3: Configuration of the double-layer FSS unit cell: (a) upper layer; (b) bottom layer; (c) the vias of the unit cell.

ECM; the results are displayed in Figure 4(b). It can be obtained that two resonant frequencies at $1.12 \mathrm{GHz}$ and $2.52 \mathrm{GHz}$ are produced when the upper layer connected with bottom layer. The frequency ranges of the dual-bandstop are $0.72-1.26 \mathrm{GHz}$ (relative bandwidth is 54.5\%) and $1.8-2.74 \mathrm{GHz}$ (relative bandwidth is $41.4 \%$ ) with the transmission coefficient lower than $-3 \mathrm{~dB}$. The length and the thickness of the double-layer FSS unit cell are about 0.037 and 0.0037 wavelength at the first resonant frequency $1.12 \mathrm{GHz}$, which means the double-layer FSS is miniaturization.
In this paper, we assume that $\theta$ is the angle between the incident wave and $z$-axis. The simulation results of the double-layer FSS with different polarizations and incidence angles are also calculated and displayed in Figure 5. It can be observed that the resonant frequencies are stable for TE and TM polarizations when $\theta=0^{\circ}$. As shown in Figure 5(a), the bandwidths widen as $\theta$ increases from $0^{\circ}$ to $60^{\circ}$ for TE polarization. The bandwidths change less when $\theta$ goes to $40^{\circ}$ and are significantly widened when $\theta=60^{\circ}$. In the meanwhile, there are a little shift by $0.9 \%$ to lower frequency and a 


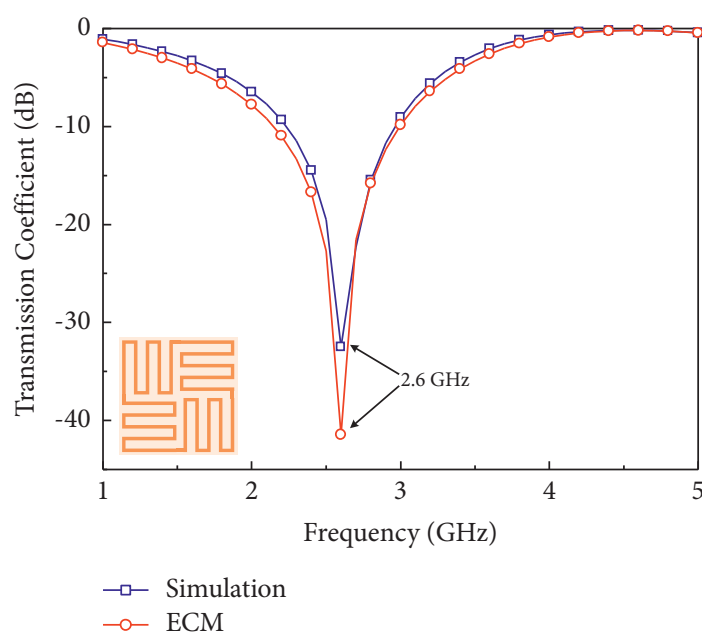

(a)

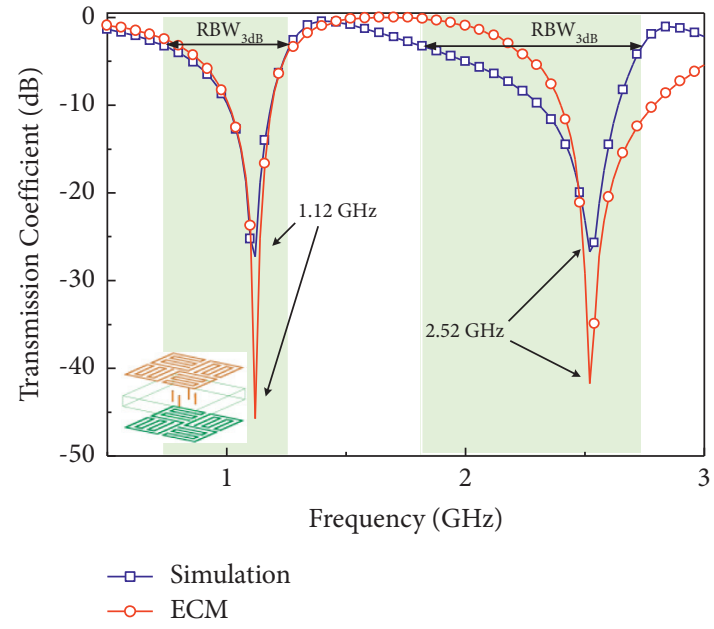

(b)

FIgURE 4: Transmission coefficient of the ECM and simulation results: (a) the single-layer FSS; (b) the double-layer FSS.

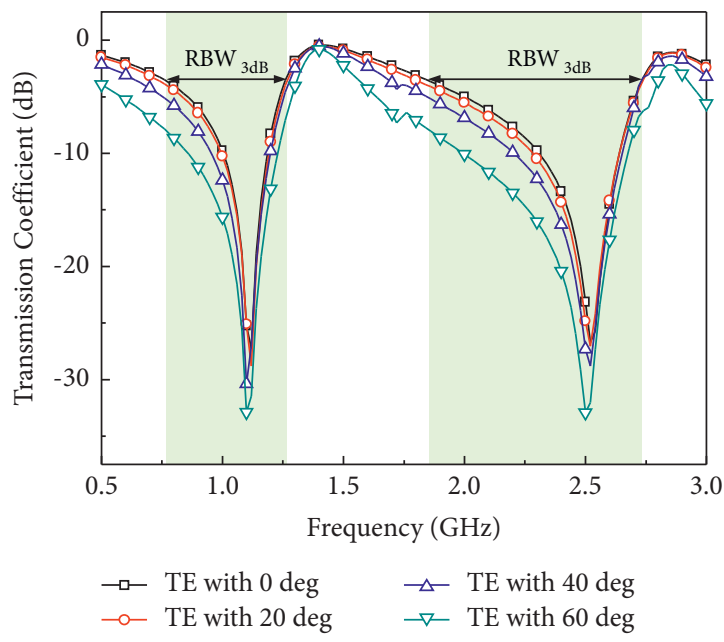

(a)

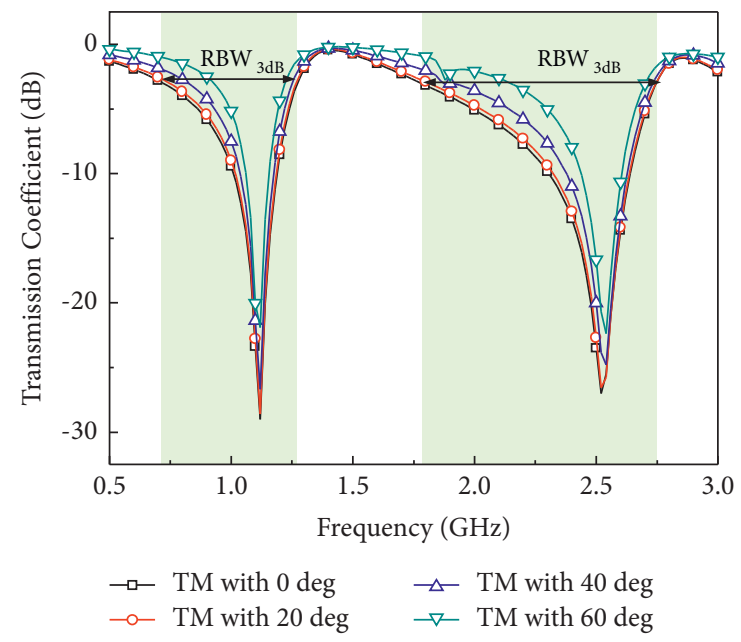

(b)

FIgURE 5: Transmission coefficients of the double-layer FSS with various $\theta$ : (a) TE polarization; (b) TM polarization.

little shift by $0.4 \%$ to higher frequency for TE polarization under $60^{\circ}$ incident angle. Conversely, the bandwidth narrows as $\theta$ increases for TM polarization as shown in Figure 5(b). The bandwidths change less when $\theta$ goes to $40^{\circ}$ and are significantly narrow when $\theta=60^{\circ}$. At the same time, there are a little shift by $0.9 \%$ to lower frequency and a little shift by $0.8 \%$ to higher frequency for TM polarization under $60^{\circ}$ incident angle. It can be observed that the double-layer FSS has a good stable performance at $\theta$ up to $40^{\circ}$.

The results shown in Figure 5 can be further explained by the surface current distributions on the proposed doublelayer FSS structure for different frequencies depicted in Figure 6. Figure 6(a) shows the current distributions on the upper and bottom layers at the first resonant frequency. The arrow in the diagram indicates the direction of the current flow. It can be observed that there are two current segments on the meander lines of the FSS structure. Figure 6(b) shows the current distributions at the second resonant frequency; the direction of the current flow indicates that there are six current segments on the meander lines. The current path is extended by connecting upper and bottom meander lines through vias, which can reduce the resonant frequencies effectively.

\section{Measurement Results}

To demonstrate the simulated performance, a prototype of the proposed double-layer FSS is fabricated and shown in Figure 7. The dimension of the fabrication is $200 \mathrm{~mm} \times 200 \mathrm{~mm}$, which contains an array of $20 \times 20$ unit cells. Two broad-band horn antennas and a vector network analyzer are used in the measurement.

As shown in Figure 8, the measured transmission coefficients under oblique incidence for both TE and TM polarizations are plotted. It can be observed that a desired dual-bandstop response is obtained, and the transmission 


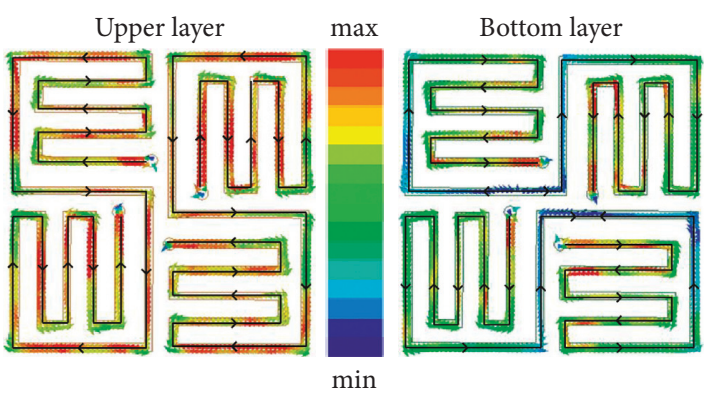

(a)

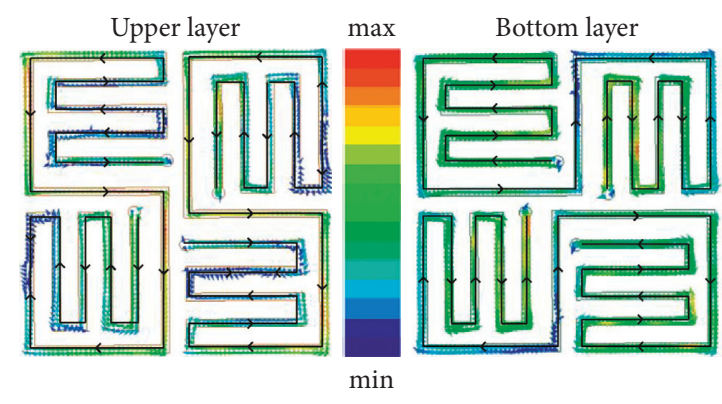

(b)

Figure 6: Surface current distributions at two resonant frequencies: (a) $1.12 \mathrm{GHz}$; (b) $2.52 \mathrm{GHz}$.

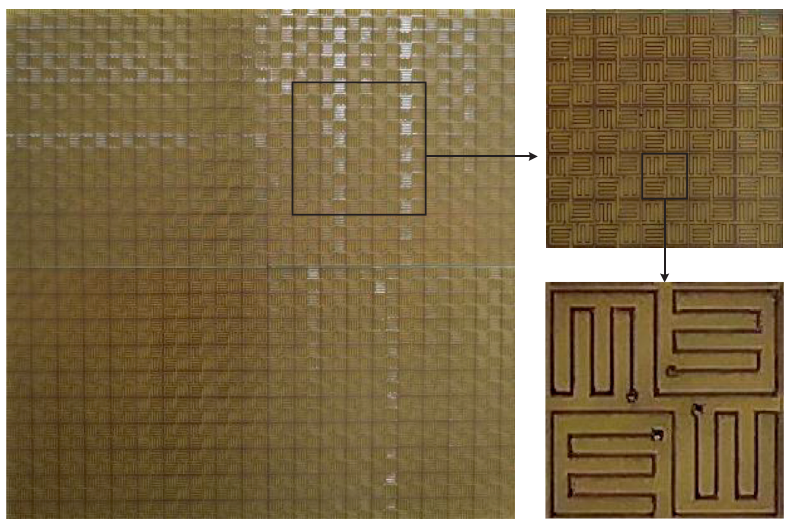

FIgURE 7: Fabricated prototype of the double-layer FSS.

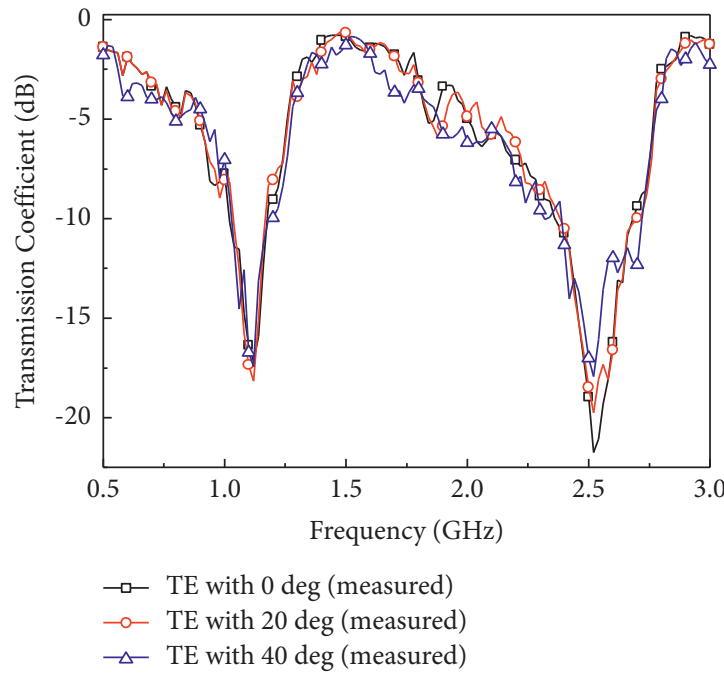

(a)

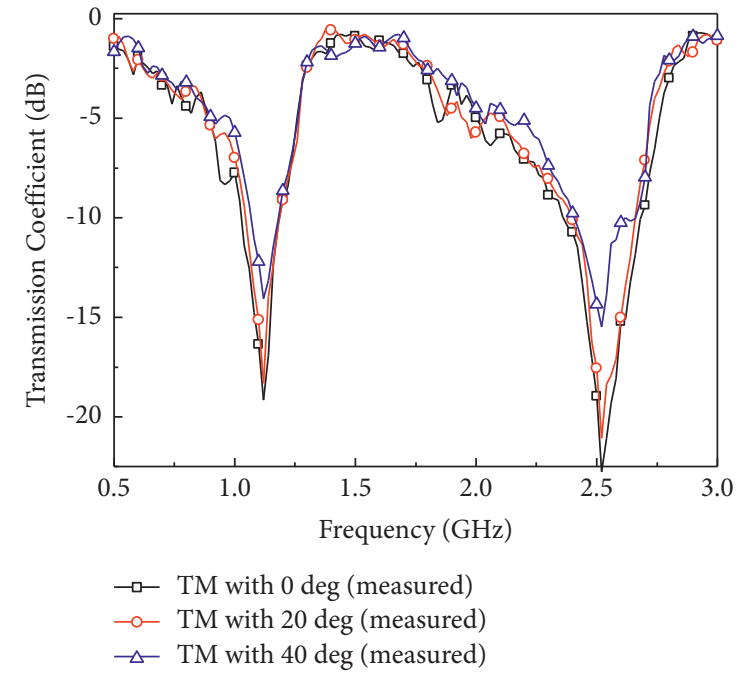

(b)

FIgURE 8: Measurement results of transmission coefficients of the dual-band FSS with various incident angles: (a) TE polarization; (b) TM polarization.

zeroes are stable under various incidence angles for both TE and TM polarizations, which are consistent with the fullwave simulations. For the normal incidence, the bandwidth of $-3 \mathrm{~dB}$ is over $0.58 \mathrm{GHz}$ and $0.98 \mathrm{GHz}$. Furthermore, the measurement results indicate that the frequency response of the proposed FSS is stable for both TE and TM polarizations, and the performance is also angular stable.

To further verify the performance of the proposed double-layer FSS, Table 1 gives the comparison of the performance between the proposed FSS design with other 
TABle 1: Performance comparison.

\begin{tabular}{|c|c|c|c|c|c|c|}
\hline Reference & Substrate thickness $(\mathrm{mm})$ & Dielectric constant & Unit cell size & Operating band & Frequency shift under $\theta$ & Polarizations \\
\hline [18] & 1.6 & 4.4 & $0.08 \lambda_{0}$ & Double & $1 \%, 60^{\circ}$ & TE, TM \\
\hline [19] & 1.6 & 4.3 & $0.066 \lambda_{0}$ & Single & $2 \%, 75^{\circ}$ & TE, TM \\
\hline [20] & 0.8 & 4.4 & $0.065 \lambda_{0}$ & Double & $0.9 \%, 75^{\circ}$ & TE, TM \\
\hline [21] & 1.6 & 4.4 & $0.052 \lambda_{0}$ & Double & $1 \%, 85^{\circ}$ & TE, TM \\
\hline$[22]$ & 0.5 & 2.65 & $0.075 \lambda_{0}$ & Double & - & TE, TM \\
\hline This work & 1 & 4.4 & $0.037 \lambda_{0}$ & Double & $0.9 \%, 60^{\circ}$ & TE, TM \\
\hline
\end{tabular}

miniaturized FSSs in the published papers. It can be observed that the unit cell size of the proposed FSS is smaller compared with other similar structures, which demonstrates that the proposed double-layer FSS is a better miniaturized design.

\section{Conclusions}

A double-layer FSS structure based on the meander lines is proposed in this paper. The application of the connection between upper and bottom layers of the structure miniaturizes the size of the unit cell effectively, which could be as small as $0.037 \lambda_{0}$ at $1.12 \mathrm{GHz}$. There are two transmission zeroes achieved by the double-layer structure, which produce the frequency response with dual-bandstop. The simulation and measurement results demonstrate that the structure is miniaturization. It is necessary to note that the resonant frequencies shift smaller than $1 \%$ for the different polarizations and the various incident angles of the plane wave, and the relative bandwidths of the structures also can be stable as the incident angle increased to $40^{\circ}$.

\section{Data Availability}

The data used to support the findings of this study are included within the article.

\section{Conflicts of Interest}

The authors declare that there are no conflicts of interest regarding the publication of this paper.

\section{Acknowledgments}

The research and publication of this article were funded by the High-Level Talent Research Starting Project of Jinling Institute of Technology (jit-b-201629).

\section{References}

[1] S. Khajevandi, H. Oraizi, and M. Poordaraee, "Design of planar dual-bandstop FSS using square-loop-enclosing superformula curves," IEEE Antennas and Wireless Propagation Letters, vol. 17, no. 5, pp. 731-734, 2018.

[2] Y. Qiang, D. Zhou, Q. Liu, and Z. Yao, "Design of low-profile frequency-selective rasorbers based on three-legged loaded element," International Journal of Antennas and Propagation, vol. 2020, p. 8, Article ID 9878607, 2020.

[3] J. Xue, W. Jiang, and S. Gong, "Wideband RCS reduction of microstrip array antenna based on absorptive frequency selective surface and microstrip resonators," International
Journal of Antennas and Propagation, vol. 2017, p. 11, Article ID 1260973, 2017.

[4] H. B. Baskey, B. Ghai, and M. J. Akhtar, "A flexible, ultra thin, frequency-selective-surface based absorber film for the radar cross section reduction of a cubical object," in Proceedings of the 2015 IEEE MTT-S International Microwave and RF Conference (IMaRC), pp. 128-131, Hyderabad, India, December 2015.

[5] S. R. Thummaluru, R. Kumar, and R. K. Chaudhary, "Isolation enhancement and radar cross section reduction of MIMO antenna with frequency selective surface," IEEE Transactions on Antennas and Propagation, vol. 66, no. 3, pp. 1595-1600, 2018.

[6] P. Zhao, Y. Zhang, R. Sun, W.-S. Zhao, Y. Hu, and G. Wang, "Design of a novel miniaturized frequency selective surface based on 2.5-dimensional jerusalem cross for 5G applications," International Journal of Antennas and Propagation, vol. 2018, p. 6, Article ID 3485208, 2018.

[7] M. Yan, S. Qu, J. Wang et al., "A miniaturized dual-band FSS with stable resonance frequencies of $2.4 \mathrm{GHz} / 5 \mathrm{GHz}$ for WLAN applications," IEEE Antennas and Wireless Propagation Letters, vol. 13, pp. 895-898, 2014.

[8] W. Yin, H. Zhang, T. Zhong, and X. Min, "A novel compact dual-band frequency selective surface for GSM shielding by utilizing a 2.5-dimensional structure," IEEE Transactions on Electromagnetic Compatibility, vol. 60, no. 6, pp. 2057-2060, 2018.

[9] S. M. A. Momeni Hasan Abadi and N. Behdad, "Design of modified single-layer double U-type linear to circular polarization convertor," International Journal of Antennas and Propagation, vol. 2019, p. 8, Article ID 1604695, 2019.

[10] U. Farooq, M. F. Shafique, and M. J. Mughal, "Polarization insensitive dual band frequency selective surface for RF shielding through glass windows," IEEE Transactions on Electromagnetic Compatibility, vol. 62, no. 1, pp. 93-100, 2020.

[11] E. Arnieri, F. Greco, and G. Amendola, "A broadband, wideangle scanning, linear-to-circular polarization converter based on standard jerusalem cross frequency selective surfaces," IEEE Transactions on Antennas and Propagation, vol. 69, no. 1, pp. 578-583, 2021.

[12] B. A. Munk, Frequency Selective Surfaces: Theory and Design, Wiley, New York, NY, USA, 2000.

[13] K. Sarabandi and N. Behdad, "A frequency selective surface with miniaturized elements," IEEE Transactions on Antennas and Propagation, vol. 55, no. 5, pp. 1239-1245, 2007.

[14] M. Al-Joumayly and N. Behdad, "A new technique for design of low-profile, second-order, bandpass frequency selective surfaces," IEEE Transactions on Antennas and Propagation, vol. 57, no. 2, pp. 452-459, 2009.

[15] T. Li, D. Li, P. Qin et al., "A novel miniaturized strongcoupled FSS structure with excellent angular stability," IEEE Transactions on Electromagnetic Compatibility, vol. 63, no. 1, pp. 38-45, 2021.

[16] W. Jiang, K. Zhang, B. Zhao, Y. Su, and S. Gong, "Design of miniaturised frequency selective rasorber using parallel LC 
resonators," IET Microwaves, Antennas \& Propagation, vol. 13, no. 5, pp. 554-558, 2019.

[17] H. Ye, J. Wei, L. Lin et al., "A frequency-selective surface rasorber based on four functional layers," IEEE Transactions on Antennas and Propagation, vol. 69, no. 5, pp. 2768-2778, 2021.

[18] C.-N. Chiu and W.-Y. Wang, "A dual-frequency miniaturized-element FSS with closely located resonances," IEEE Antennas and Wireless Propagation Letters, vol. 12, pp. 163$165,2013$.

[19] S. Peddakrishna, T. Khan, B. K. Kanaujia, and N. Nasimuddin, "Design of a compact passband frequency selective surface with stable resonance," International Journal of Antennas and Propagation, vol. 2017, p. 5, Article ID 7696039, 2017.

[20] S. Ghosh and K. V. Srivastava, "An angularly stable dual-band FSS with closely spaced resonances using miniaturized unit cell," IEEE Microwave and Wireless Components Letters, vol. 27, no. 3, pp. 218-220, 2017.

[21] P.-S. Wei, C.-N. Chiu, C.-C. Chou, and T.-L. Wu, "Miniaturized dual-band FSS suitable for curved surface application," IEEE Antennas and Wireless Propagation Letters, vol. 19, no. 12, pp. 2265-2269, 2020.

[22] N. Liu, X. Sheng, X. Gao, C. Zhang, and D. Guo, "Design of dual-band miniaturized frequency selective surface using branched tortuous structure," ACES Journal, vol. 34, no. 1, pp. 49-55, 2019.

[23] F. Huo, J. Bao, M. Zhu, F. Liu, and Y. Tao, "Design of miniaturized dual-band frequency selective surface with spiral arrangement of meander lines," IEICE Electronics Express, vol. 18, no. 20, pp. 1-5, 2021.

[24] Y. Ma, W. Wu, Y. Yuan, X. Zhang, and N. Yuan, "A convoluted structure for miniaturized dual-bandstop frequency selective surface," IEEE Antennas and Wireless Propagation Letters, vol. 18, no. 2, pp. 328-332, 2019. 